\title{
Prenatal predictors of objectively measured appetite regulation in low-income toddlers and preschool-age children
}

\author{
Janne Boone-Heinonen ${ }^{1}$ (D) | Heidi M. Weeks ${ }^{2}$ (D) | Julie Sturza ${ }^{3}$ | Alison L. Miller ${ }^{4,5}$ | \\ Julie C. Lumeng ${ }^{2,3,4}$ | Katherine W. Bauer ${ }^{2,4}$
}

\author{
${ }^{1}$ School of Public Health, Oregon Health \& \\ Science University, Portland, Oregon, USA \\ ${ }^{2}$ Department of Nutritional Sciences, \\ University of Michigan School of Public Health, \\ University of Michigan, Ann Arbor, Michigan, \\ USA \\ ${ }^{3}$ Department of Pediatrics, University of \\ Michigan Medical School, Ann Arbor, \\ Michigan, USA \\ ${ }^{4}$ Center for Human Growth and Development, \\ University of Michigan, Ann Arbor, Michigan, \\ USA \\ ${ }^{5}$ Department of Health Behavior and Health \\ Education, University of Michigan School of \\ Public Health, University of Michigan, Ann \\ Arbor, Michigan, USA \\ Correspondence \\ Janne Boone-Heinonen, School of Public \\ Health, Oregon Health \& Science University, \\ 3181 SW Sam Jackson Park Rd, CB669, \\ Portland, OR 97239, USA. \\ Email: boonej@ohsu.edu \\ Funding information \\ National Institute of Diabetes and Digestive \\ and Kidney Diseases, Grant/Award Number: \\ K01DK102857; Eunice Kennedy Shriver \\ National Institute of Child Health and Human \\ Development, Grant/Award Number: \\ R01HD069179
}

\author{
Summary \\ Background: Prenatal nutrition impacts offspring appetite regulation in animal \\ models. However, evidence from humans is scarce. \\ Objective: To determine associations between indicators of prenatal nutrition and \\ appetite regulation among young children.
}

Methods: Participants included 454 low-income mother/child dyads (mean child age $=45.2$ months $[S D=9.7]$ ). Children's appetite regulation was ascertained with the maternal-reported Child Eating Behavior Questionnaire and objectively assessed using the Eating in the Absence of Hunger protocol. Using hierarchical linear regression, we modelled child appetite regulation measures as a function of prenatal nutrition indicators (child birthweight $\mathrm{z}$ scores $\left[\mathrm{BWz}, \mathrm{BW} \mathrm{z}^{2}\right]$; maternal prepregnancy body mass index [BMI], gestational weight gain [GWG]), adjusted for sociodemographic characteristics.

Results: Among girls, higher and lower birthweight were associated with greater energy consumed in the absence of hunger, primarily sweet foods, coeff $(95 \% \mathrm{Cl})$ : BWz $0.17(0.05,0.28), \mathrm{BWz}^{2} 0.15(0.04,0.26)$, but not food responsiveness or food enjoyment. Higher birthweight was also associated with greater satiety responsiveness among girls. Among boys, birthweight was unrelated to measures of appetite regulation. Associations between maternal BMI and GWG and child appetite regulation were inconsistent.

Conclusions: Among low-income girls, but not boys, indicators of adverse prenatal conditions were associated with poor objectively measured appetite regulation during early childhood.

\section{KEYWORDS}

appetite regulation, birthweight, early childhood risk factors, maternal obesity

\section{1 | INTRODUCTION}

In the United States, the prevalence of childhood obesity remains high with no signs of decline: $26 \%$ of children have obesity and $7.9 \%$ have severe obesity. ${ }^{1}$ In the current food environment, individuals with a poorer ability to self-regulate energy intake are vulnerable to overconsumption, particularly of highly palatable, energy-dense foods, resulting in excess weight gain. ${ }^{2}$ Therefore, identifying modifiable factors that disrupt appetite regulation is a public health priority. Growing evidence suggests that exposures during the prenatal period, such as maternal diet, may influence the development of neuronal and hormonal factors involved in offspring appetite regulation., ${ }^{3,4}$ Poor 
appetite regulation therefore may be a critical mechanism underlying developmentally induced predisposition to obesity. ${ }^{5}$ However, human evidence is limited.

In animal models, both foetal undernutrition and overnutrition induces elevated appetite, preferences for foods high in sugar and fat, and subsequent obesity. ${ }^{6}$ Mechanisms underlying effects on appetite regulation include alterations to the hypothalamic appetiteregulating network, nutrient sensing, and neuronal development and leptin and insulin signalling. ${ }^{7}$ Human evidence that prenatal exposures impact appetite regulation is critical for understanding these processes in the context of environmental and genetic variability that exists in human populations ${ }^{8,9}$ but is, to date, limited and focused on foetal undernutrition. Measures of appetite regulation can be broadly classified as food avoidant behaviours such as picky eating or food refusal and food approach behaviours such as low satiety response, which are associated with later obesity. ${ }^{10}$ In studies using parent-reported child eating behaviours, low birthweight (LBW) or intrauterine growth restriction (IUGR) is associated with greater eating in response to external food cues, strength of attempts to restrain eating, ${ }^{11}$ and food avoidant behaviours. ${ }^{12,13}$ Among the even smaller number of studies to examine objectively measured appetite regulation, young children born with IUGR (compared with those with normal birth size) have demonstrated greater impulsive eating ascertained with a snack delay test. ${ }^{14}$ Similarly, adolescents born with lower birthweight experience elevated neural activation in response to viewing palatable foods. ${ }^{11}$ In summary, human evidence is consistent with mechanistic understanding of prenatal influences on neurocircuitry that drive appetite regulation but remains small, focused on children born with LBW, based outside of the United States, and largely based on parentreported eating behaviours.

Meanwhile, while maternal obesity is consistently associated with up to a threefold greater risk of obesity in the next generation, ${ }^{15}$ evidence for the influence of foetal overnutrition on appetite regulation among human offspring is scant. The few studies that examine higher birthweight in relation to child appetite regulation focus on food avoidant behaviours, ${ }^{12,13}$ as opposed to food approach behaviours, despite the strong linkage between food approach behaviours and high risk for obesity. Children born large for gestational age tend to be less likely to be perceived by parents as poor eaters (eating small or insufficient quantity) $)^{12}$ and less likely to exhibit persistent picky eating ${ }^{13}$ but otherwise show no clear patterns in differences in food avoidant behaviours. ${ }^{12}$ In one recent US study, girls, but not boys, exposed prenatally to impaired maternal glucose tolerance exhibited elevated eating in the absence of hunger in early adolescence, ${ }^{16}$ a manifestation of strong food approach tendencies, but when such changes emerge in childhood has not been studied.

Knowledge of the impact of both foetal undernutrition and overnutrition on appetite regulation offers the potential to identify intervention targets for the primary and secondary prevention of childhood obesity and later development of cardiometabolic disease. ${ }^{17}$ Therefore, the objective of this study was to test the hypothesis that indicators of foetal undernutrition and overnutrition (higher or lower birthweight, higher maternal pre-pregnancy body mass index [BMI], and excessive gestational weight gain [GWG]) are associated with objectively measured and parent-reported food approach behaviours among a sample of low-income young children.

\section{2 | METHODS}

Data were obtained from two Appetite, Behavior, and Cortisol (ABC) cohorts of low-income parent/child dyads recruited from southcentral Michigan: ABC Preschool and ABC Toddler. In ABC Preschool, parents and their 3 to 5 -year-old children were recruited between 2009 and 2011 based on their participation in Head Start, the federally supported programme that provides preschool to children with household incomes at or below the federal poverty line. To be eligible for $A B C$ Preschool, parents had to be fluent in English and have less than a 4-year college degree. Children who were younger than 35 weeks gestational age at birth; had significant perinatal or neonatal complications, serious medical problems, or food allergies; or in foster care were excluded from recruitment into the $A B C$ studies.

$A B C$ Toddler aimed to enrol a demographically similar cohort of low-income parent/child dyads as ABC Preschool. ABC Toddler recruited biological mothers and their toddler-aged (21-27 months) children between 2011 and 2014 through flyers posted in community agencies serving low-income families. With the exception of only recruiting children's biological mothers, inclusion and exclusion criteria for $A B C$ Toddler and $A B C$ Preschool were identical. Data from the toddlers' 33-month visit were used in the current study.

For the current study, dyads in the ABC studies were excluded if the parent participating was not the child's biological mother $(n=27)$ or data on parental relationship were missing $(N=22)$, the child's gestational age at birth was 37 weeks or younger or data were missing $(\mathrm{N}=55)$, or the dyad was missing all measures of eating behaviour $(\mathrm{N}=61)$ or all measures of perinatal exposures $(\mathrm{N}=5)$. Preterm births (37 weeks or less) were excluded from this analysis to minimize the influence of feeding difficulties resulting from prematurity. ${ }^{18,19}$ The final study sample included 454 mother/child dyads, 307 from $A B C$ Preschool and 147 from $A B C$ Toddler. This represents $72.8 \%$ of the total 624 dyads from these studies. All mothers provided written informed consent, and the University of Michigan Institutional Review Board approved the studies.

\section{$2.1 \mid$ Measures}

Measures were conducted with mother/child dyads during study visits conducted by trained research staff either in community settings or in the dyads' homes.

\subsection{1 | Prenatal characteristics}

Mothers reported their child's due date, birthdate, and birthweight. The child's gestational age at birth was calculated by comparing their due date with birthdate. Child birthweight $z$ scores (BWz) accounting for gestational age were calculated using nationally representative 
reference values identified by Oken et $\mathrm{al}^{20}$; then, for descriptive purposes, these were classified as small (SGA, less than 10th percentile), appropriate (AGA), and large (LGA; greater than 90th percentile). Mothers self-reported their pre-pregnancy weight, shown in other studies to be reliable 2 to 5 years after birth. ${ }^{21}$ Mothers' height was either measured using standardized protocols by trained research assistants during the study visit $(\mathrm{N}=487)$ or self-reported $(\mathrm{N}=4)$. Pre-pregnancy BMI was then calculated from these measures. Mothers also reported their GWG, shown in prior work to correlate $(r=0.63)$ with documented GWG. ${ }^{22}$ GWG was categorized as insufficient, adequate, and excessive based on the 2009 Institute of Medicine's pre-pregnancy weight status-specific guidelines: underweight (28-40 pounds), normal weight (25-35 pounds), overweight (15-25 pounds), and obesity (11-20 pounds). ${ }^{23}$

\subsection{2 | Child appetite regulation measures}

Appetite regulation was ascertained via mother-report on the Children's Eating Behavior Questionnaire (CEBQ) and objectively assessed by children's measured intake during the Eating in the Absence of Hunger (EAH) protocol, a measure of appetite regulation that reflects children's consumption of highly palatable snack foods after a satiating meal. The CEBQ is a 35-item instrument consisting of eight subscales of children's eating behaviours; for all subscales, mothers rate their child's eating behaviours on 5-point Likert scales ranging from Never (1) to Always (5); higher scores indicate greater endorsement of the eating behaviour. ${ }^{24}$ Three CEBQ subscales reflecting food approach behaviours were included in the current study based on their previous consistent associations with child obesity risk: enjoyment of food, which assesses a mother's perception of her child's desire to eat and enjoyment of food (four items, Cronbach's $\alpha=.80$ ); food responsiveness (eating in response to environmental food cues; five items, Cronbach's $a=.78$ ); and satiety responsiveness (attention to internal cues of fullness; five items, Cronbach's $a=.73$ ). ${ }^{25,26}$

The EAH protocols used with children in $A B C$ Preschool and Toddler cohorts have been described in detail previously. ${ }^{27,28}$ In ABC Preschool, children's EAH was measured immediately after breakfast at Head Start. Following breakfast, the research assistant determined the child's satiation using cartoon figures and proceeded with engaging the child in the EAH task only if the child reported that they were not hungry. The research assistant then invited the child to a separate room and for 10 minutes gave them free access to toys and premeasured bowls of Trix cereal (32 grams, 120 calories), mini Chips Ahoy cookies (90 g, 435 kcal), fruit snacks (164 g, 631 kcal), Cheez-lts (60 g, 300 kcal), pretzel sticks (36 g, 129 kcal), and Chicken in a Biskit crackers ( $60 \mathrm{~g}, 310 \mathrm{kcal})$. Children were told "You can play with any of the toys and eat any of the foods on this table. I'm going to do some work." After 10 minutes, the food remaining was weighed to determine the amount of each food children consumed and the corresponding energy intake. Higher energy consumption represents a higher degree of EAH.

Minor modifications to the EAH protocol was used during $A B C$ Toddler given the developmental capacity of toddlers. Briefly, the protocol was conducted in dyads' homes, and mothers were asked to have their children fast for 1 hour and then served a typical lunch. After lunch, the research assistant presented a standardized plate of highly palatable, developmentally appropriate sweet and salty snack foods (Nabisco Original Chips Ahoy chocolate chip cookies: $22.0 \mathrm{~g}$, 106.4 kcal; Nabisco Original Oreo cookies: 23.2 g, 109.3 kcal; Keebler Animal Cookies, Frosted: 19.0 g, 97.9 kcal; Nabisco Rainbow Candy Blast Chips Ahoy cookies: 33.4 g, 176.8 kcal; Kellogg's Keebler Fudge Stripe chocolate-coated cookies: 23.6 g, 122.1 kcal; Pringles potato chips: 18.2 g, 97.6 kcal; Frito-Lay Cheetos cheese puffs: 20.3 g; $108.8 \mathrm{kcal})$. Research assistants then told the child "Here are some special treats you can eat," and to signal to the toddler that they could eat the foods offered, the research assistant ate one cookie off the plate and said, "I'm going to have one, too. Mmm this is really good. You can eat as much as you want." The child was then given free access to the food. The mother was asked not to interact with the child during the protocol to minimize external prompts to eat. After 10 minutes, the plate of food was removed. Remaining food was weighed and kcal of each food consumed were calculated.

Because the protocol, including foods offered to children, varied between the cohorts, children's total kcal intake and intake of sweet and salty foods were standardized within each cohort to a mean of 0 and SD of 1 and then combined across the cohorts.

\subsection{3 | Sociodemographic and other characteristics}

Mothers reported sociodemographic characteristics for herself and her child including the child's sex, race, and ethnicity and her own educational attainment. Child race/ethnicity included non-Hispanic white, black, biracial, or other and Hispanic; because of small cell sizes, race/ethnicity was analysed as non-Hispanic white versus other in multivariable analysis. Children's age at the time of data collection was calculated from their birthdate. Children's height and weight were measured using standardized protocols by trained research assistants during the study visit. Using the 2000 CDC growth charts, ${ }^{29}$ sexand age-specific BMI $z$ scores and percentiles were calculated. On the basis of the BMI-for-age percentiles, child BMI was classified as underweight (less than 5th), normal weight (greater than or equal to 5 th to less than 85 th), overweight (greater than or equal to 85 th to less than 95th), or obesity (greater than or equal to 95th).

\section{2 | Statistical analysis}

All analyses were conducted using SAS 9.4. All analyses were stratified by sex, recognizing previously described sex differences in prenatal development indicators and obesity-related outcomes. ${ }^{7,30}$ Linear regression was used to model each eating behaviour as a function of one of three prenatal development indicators (BWz, maternal prepregnancy $\mathrm{BMI}$, or GWG) and a priori confounders (BWz: maternal education and race/ethnicity; maternal BMI: maternal education, race/ethnicity, and age; and GWG: maternal education, race/ethnicity, age, and pre-pregnancy BMI). Nonlinear associations with continuous independent variables (BWz and maternal $\mathrm{BMI}$ ) were assessed by 
testing for quadratic and cubic terms (Wald significance of the quadratic or cubic term $P<.10)$. BWz quadratic terms were significant in several models and retained, for comparability, for all models. The BWz cubic term was significant and retained for satiety responsiveness in girls. Statistical significance was assessed the $95 \%$ confidence level.

\section{3 | RESULTS}

Data from 222 girls and 232 boys were included in the current study. Over $40 \%$ of mothers had a high school or less education, and $45 \%$ to $46 \%$ were from minority racial/ethnic groups (Table 1). Mean BWz was -0.17 for girls and -0.25 for boys, with variation across the birthweight spectrum: SGA prevalence was $14.2 \%$ in girls and $13.3 \%$ in boys; LGA prevalence was $9.5 \%$ in girls and $4.6 \%$ in boys. Mean maternal pre-pregnancy BMI was in the overweight range (approximately $28 \mathrm{~kg} \mathrm{~m}^{-2}$ ), and over half of mothers gained more weight than recommended during pregnancy. Among girls, 38.5\% had overweight or obesity, and $32.0 \%$ of boys had overweight or obesity. Girls and boys consumed a mean of 77.4 and $101.0 \mathrm{kcal}$ in the absence of hunger, respectively, approximately $80 \%$ from sweet foods. Sweet and total foods eaten in the absence of hunger were weakly correlated with food responsiveness (Spearman: 0.17 and 0.18 , respectively) and uncorrelated with enjoyment of food (Spearman: 0.03 and 0.04) and satiety responsiveness (Spearman: -0.06 and -0.04 ) (data not shown).

In adjusted analysis, girls born with lower or higher BWz consumed more kilocalories from sweet foods and, correspondingly, total kilocalories, in the absence of hunger than girls with moderate BWz (Table 2; Figure 1). Also among girls, BWz exhibited U-shaped associations with parent-reported food responsiveness and enjoyment of food, although these associations were not statistically significant; the cubic association with satiety responsiveness exhibited lower satiety responsiveness for lower BWz, moderate satiety responsiveness in the normal range of $\mathrm{BWz}$, and higher satiety responsiveness in the higher range of BWz. Higher maternal pre-pregnancy BMI was associated with significantly higher food responsiveness and enjoyment of food (Table 2). GWG was not associated with appetite regulation in girls.

In boys, prenatal predictors were, in general, not related to measures of appetite regulation (Table 3; Figure 1). In two exceptions, higher maternal pre-pregnancy BMI was associated with higher satiety responsiveness, and less than recommended GWG was associated with lower satiety responsiveness in the child.

\section{4 | DISCUSSION}

Among low-income toddler and preschool-aged girls, but not boys, both higher and lower BWz were associated with greater energy consumed in the absence of hunger, particularly from sweet foods. Eating in the absence of hunger among children is indicative of poor appetite regulation and predictive of excess weight gain and obesity. ${ }^{27,31,32}$ Further, also among girls, lower BWz was associated with lower satiety responsiveness, another eating behaviour that places children at increased risk for obesity, ${ }^{33,34}$ while higher BWz was associated with higher satiety responsiveness. In contrast, associations between maternal BMI and GWG with child appetite regulation were inconsistent. Together, these findings partially support the hypothesis that adverse prenatal conditions are associated with poor appetite regulation during early childhood but suggest variation across food approach behaviours. Further, some exposures (eg, factors that lead to higher infant birthweight) may be more important than others (eg, maternal pre-pregnancy weight status) in altering children's appetite regulation. Finally, the lack of similar associations observed among boys also suggest sex differences in the potential impacts of the prenatal environment factors that influence child growth, as has been observed in previous research. ${ }^{7,30}$

The observed associations between lower BWz and greater eating in the absence of hunger among girls are consistent with prior evidence that IUGR and LBW are associated with greater external and restrained eating, neuronal response to viewing of palatable foods in children, ${ }^{11}$ objectively assessed impulsive eating in girls, ${ }^{14}$ and sweet taste preference. ${ }^{35}$ This study contributes to this growing but still small body of evidence by demonstrating associations between lower birthweight and an objective measure of appetite regulation. These eating behaviours may serve to explain associations between low birthweight and risk for cardiometabolic disease ${ }^{4}$ and, in some study populations, obesity. ${ }^{36}$

With regard to the observed sex differences, prior works suggest that developmental programming occurs in a sex-dependent manner, ${ }^{37-39}$ yet sex differences in birthweight-eating behaviour associations are mixed. Silveria and colleagues found associations between IUGR and impulsive eating in girls but not in boys, ${ }^{14}$ while Van Deutokem et al found similar associations between birthweight and satiety responsiveness in boys and girls ${ }^{40}$; yet, most studies lacked sufficient sample size to examine sex differences. Further research on mechanisms underlying sex-specific associations between birthweight and appetite regulation is needed.

Our study adds to the extant literature regarding birthweight and child appetite regulation by providing some of the first evidence for associations across the full spectrum of birthweight and child eating behaviour, including large birth size (LGA or high birthweight), which is strongly associated with later obesity. ${ }^{41} \mathrm{~A}$ small number of studies examine birthweight as a continuous variable, finding less picky eating and less neuronal response to food with increasing birthweight. ${ }^{11,13}$ Yet, consideration of only linear associations may distort associations by precluding identification of adverse outcomes at both the lower and higher ends of the spectrum of birth size. Oliveira and colleagues examined LGA births, finding mixed associations suggestive of lower risk of maternal-reported food avoidant eating behaviours. ${ }^{12}$ However, the study did not examine food approach behaviours. In girls, our findings that higher birthweight is associated with greater satiety responsiveness are counter to expectation and may reflect differences between objectively measured and parent-reported appetite regulation or differences in specific dimensions of food approach behaviour (eg, food responsiveness and satiety responsiveness) that may drive 
TABLE 1 Characteristics of mother/child dyads in the ABC Study by child sex

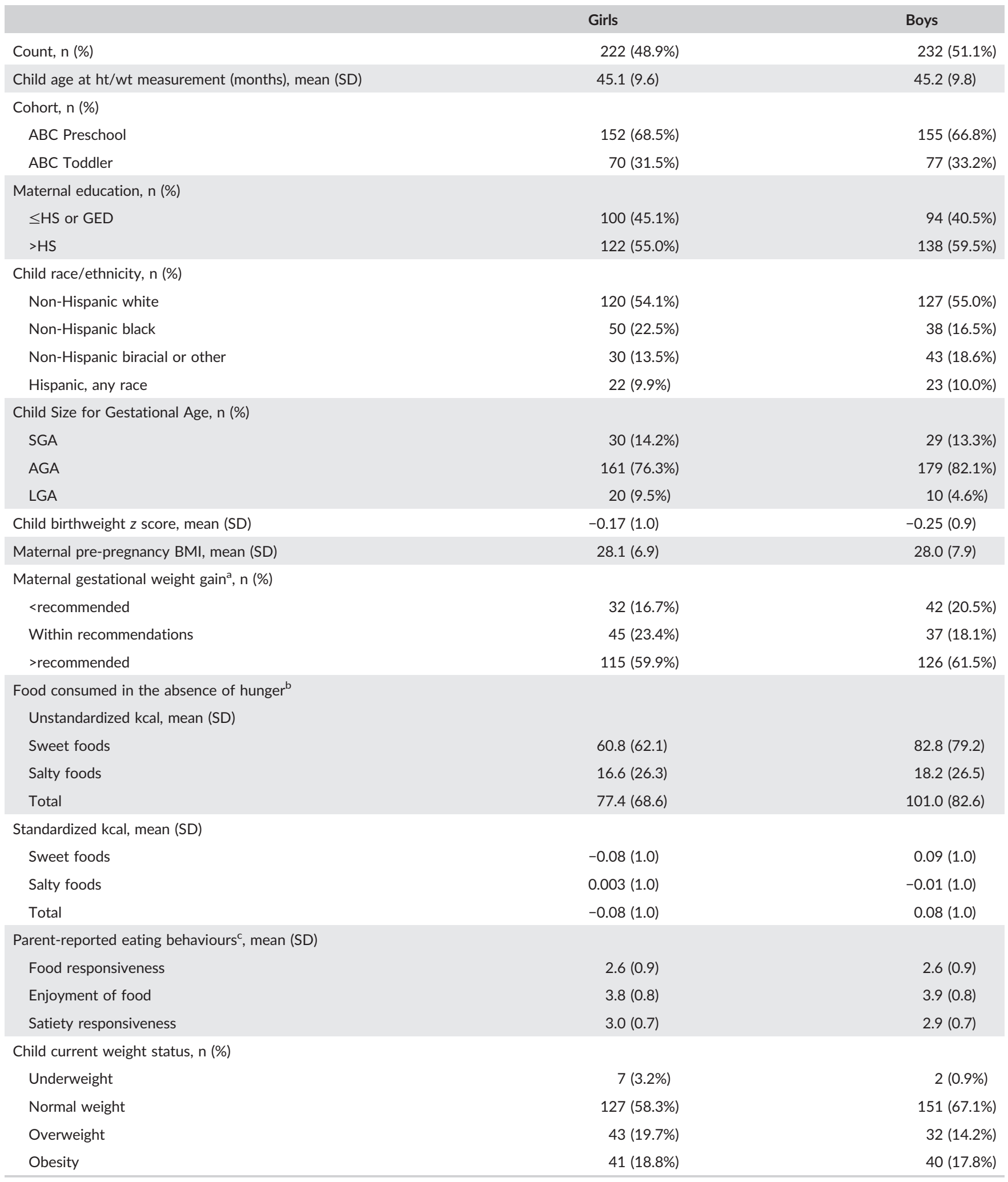

Abbreviations: ABC, Appetite, Behavior, and Cortisol; AGA, appropriate for gestational age; BMI, body mass index; GED, general educational development; HS, high school; LGA, large for gestational age; SGA, small for gestational age.

${ }^{a}$ Gestational weight gain (GWG) categories are relative to Institute of Medicine 2009 guidelines: pre-pregnancy underweight (28-40 pounds), normal weight (25-35 pounds), overweight (15-25 pounds), and obesity (11-20 pounds).

${ }^{\mathrm{b}}$ Ascertained from the objective Eating in the Absence of Hunger protocol.

'Ascertained from the Children's Eating Behavior Questionnaire. 
TABLE 2 Association of indicators of adverse prenatal conditions and eating behaviours in girls ${ }^{a}$

\begin{tabular}{|c|c|c|c|c|c|}
\hline & \multicolumn{2}{|c|}{ Food Eaten in the Absence of Hunger ${ }^{b}$} & \multicolumn{3}{|l|}{ Parent-Reported ${ }^{\mathrm{C}}$} \\
\hline & Sweet & Total & Food Responsiveness & Enjoyment of Food & Satiety Responsiveness \\
\hline \multicolumn{6}{|l|}{ Birthweight $z$ score (BWz) } \\
\hline $\mathrm{BWz}^{2}$ & $0.17(0.05,0.28)$ & $0.15(0.04,0.26)$ & $0.08(-0.01,0.17)$ & $0.06(-0.03,0.14)$ & $-0.04(-0.12,0.04)$ \\
\hline $\mathrm{BW} \mathrm{z}^{3}$ & - & - & - & - & $0.07(0.02,0.13)$ \\
\hline \multicolumn{6}{|l|}{ Gestational weight gain } \\
\hline$<$ recommended & $-0.14(-0.60,0.32)$ & $-0.30(-0.76,0.16)$ & $-0.01(-0.42,0.40)$ & $-0.01(-0.40,0.37)$ & $0.03(-0.30,0.37)$ \\
\hline >recommended & $-0.03(-0.39,0.32)$ & $-0.03(-0.38,0.33)$ & $0.03(-0.29,0.35)$ & $0.07(-0.23,0.36)$ & $-0.11(-0.37,0.15)$ \\
\hline
\end{tabular}

Toddler and preschool-age girls in the Appetite, Behavior, and Cortisol (ABC) Study. Estimates obtained from sex-stratified linear regression modelling a single eating behaviour as a function of birthweight $z$ score $\left(B W z\right.$; linear $(B W z)$ and quadratic $\left(B W z^{2}\right)$ and, for satiety responsiveness cubic $\left.\left(B W z^{3}\right)\right)$, maternal pre-pregnancy BMI (linear), or gestational weight gain (GWG) relative to Institute of Medicine 2009 guidelines; control variables (birthweight: maternal education and race/ethnicity; maternal BMI: maternal education, race/ethnicity, and age; GWG: maternal education, race/ethnicity, age, and pre-pregnancy BMI).

${ }^{b}$ Objectively measured eating behaviours ascertained from the Eating in the Absence of Hunger protocol $(n=195$ for birthweight, $n=184$ for maternal BMI, $n=177$ for GWG). Associations for salty foods eaten in the absence of hunger are not reported because of the small portions consumed (Table 1). 'Parent-reported eating behaviours ascertained from the Children's Eating Behavior Questionnaire ( $\mathrm{n}=201$ for birthweight, $\mathrm{n}=188 \mathrm{for}$ maternal BMI, $\mathrm{n}=181$ for GWG)

Girls
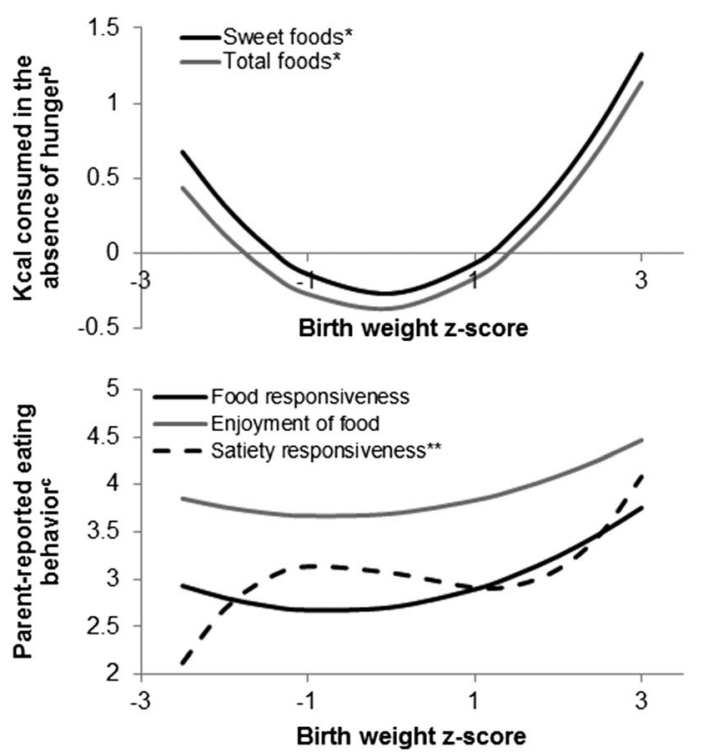

Boys
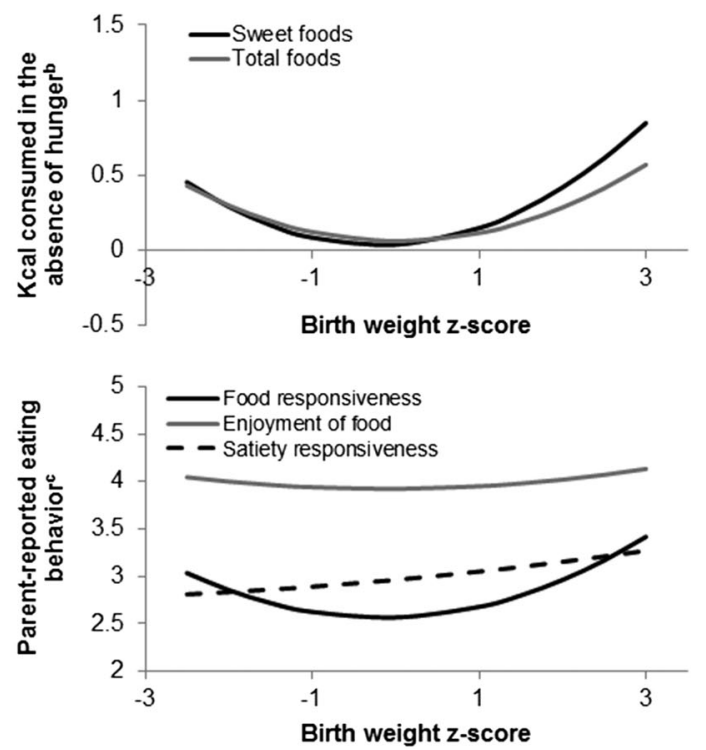

FIGURE 1 Association of birthweight $z$ score and eating behaviours in girls and boys ${ }^{\text {a }}$. Toddler and preschool-age girls in the Appetite, Behavior, and Cortisol (ABC) Study. Estimates obtained from sex-stratified linear regression modelling a single appetitive trait as a function of birthweight $z$ score and control variables (maternal education and race/ethnicity). ${ }^{\mathrm{b}}$ Objectively measured appetitive traits ascertained from the Eating in the Absence of Hunger protocol $(n=195)$. 'Parent-reported appetitive traits ascertained from the Children's Eating Behavior Questionnaire ( $n=201)$. *Quadratic birthweight $z$ score term is significant (Wald $P<.1)$. ${ }^{* *}$ Cubic birthweight $z$ score term is significant $($ Wald $P<.1)$

eating in the absence of hunger. Influences of specific prenatal exposures with multiple dimensions of food approach behaviour in both girls and boys offer an important area for future research.

The current study's largely null associations between prepregnancy BMI or GWG and children's food approach behaviour are inconsistent with extensive animal evidence that diet-induced maternal obesity alters neurocircuitry in the offspring that increases appetite and food intake. ${ }^{6,7}$ These effects are observed during both the prenatal and postnatal period, reflecting effects that occur through intrauterine mechanisms and lactation. Our findings likely reflect greater complexity of appetite regulation in humans, involving environmental, psychosocial, and genetic drivers that are an active area of research. ${ }^{8,10}$ Additionally, we were not able to examine associations between postnatal factors (eg, lactation) and eating behaviours. It is 
TABLE 3 Association of indicators of adverse prenatal conditions and eating behaviours in boys ${ }^{\mathrm{a}}$

\begin{tabular}{|c|c|c|c|c|c|}
\hline & \multicolumn{2}{|c|}{ Food Eaten in the Absence of Hunger ${ }^{b}$} & \multicolumn{3}{|l|}{ Parent-Reported ${ }^{c}$} \\
\hline & Sweet & Total & Food Responsiveness & Enjoyment of Food & Satiety Responsiveness \\
\hline \multicolumn{6}{|l|}{ Birthweight $z$ score } \\
\hline$B W z^{2}$ & $0.08(-0.07,0.22)$ & $0.06(-0.09,0.20)$ & $0.09(-0.04,0.21)$ & $0.02(-0.08,0.12)$ & $0.01(-0.08,0.10)$ \\
\hline Maternal body mass index & $0.01(0.00,0.03)$ & $0.01(-0.01,0.03)$ & $0.00(-0.02,0.02)$ & $0.00(-0.02,0.01)$ & $0.02(0.01,0.03)$ \\
\hline$<$ recommended & $-0.02(-0.48,0.45)$ & $-0.18(-0.66,0.30)$ & $0.04(-0.39,0.47)$ & $0.16(-0.20,0.51)$ & $-0.34(-0.65,-0.04)$ \\
\hline >recommended & $-0.28(-0.67,0.11)$ & $-0.29(-0.69,0.11)$ & $0.03(-0.32,0.39)$ & $0.06(-0.24,0.36)$ & $-0.15(-0.41,0.11)$ \\
\hline
\end{tabular}

Toddler and preschool-age boys in the Appetite, Behavior, and Cortisol (ABC) Study. Estimates obtained from sex-stratified linear regression modelling a single eating behaviour as a function of birthweight $z$ score (BWz), maternal pre-pregnancy BMI (linear), or gestational weight gain (GWG) relative to Institute of Medicine 2009 guidelines; control variables (birthweight: maternal education and race/ethnicity; maternal BMI: maternal education, race/ethnicity, and age; GWG:maternal education, race/ethnicity, age, and pre-pregnancy BMI).

bObjectively measured eating behaviours ascertained from the Eating in the Absence of Hunger protocol $(n=203$ for birthweight, $n=200$ for maternal BMI, $n=189$ for GWG). Associations for Salty foods eaten in the absence of hunger are not reported because of the small portions consumed (Table 1).

'Parent-reported eating behaviours ascertained from the Children's Eating Behavior Questionnaire ( $\mathrm{n}=212$ for birthweight, $\mathrm{n}=209$ for maternal BMI, $\mathrm{n}=$ 198 for GWG)

also possible that prenatal influences on appetite regulation manifest later in childhood.

Our findings suggest that poor appetite regulation may be a mechanism through which intrauterine programming promotes obesity risk while guiding areas in need of additional research. First, greater understanding of the independent and joint roles of prenatal and infant growth on appetite regulation is needed. ${ }^{40}$ Second, the role of prenatal determinants on distinct aspects of appetite regulation-for example, enjoyment of food or satiety responsiveness-requires further study. Third, the contributions of genetic and ongoing environmental factors that influence both prenatal growth and postnatal development of appetite regulation is needed. For example, shared genetic variants are associated with maternal weight status and prenatal and postnatal growth, ${ }^{42}$ potentially through appetite regulation. Further research with in-depth characterization of maternal pre-pregnancy, prenatal, and child characteristics can help inform our causal understanding of the mechanisms by which the prenatal environment impacts children's eating and growth.

\subsection{Limitations and strengths}

Findings from this study should be interpreted in the context of several limitations. First, our exposures of interest-BWz, maternal pre-pregnancy BMI, and GWG-were calculated using data retrospectively reported by mothers. While maternal report of birthweight has been shown to be valid in prior research, there is variation in body composition at any given BMI. ${ }^{43}$ Self-reported pre-pregnancy weight and GWG are also known to have errors, including misclassification error of GWG, potentially attenuating the observed associations. ${ }^{22,44}$ Second, we lacked measures of eating behaviours during infancy, precluding investigation of the emergence of poor appetite regulation during early childhood or the extent to which adiposity during infancy played a role in toddler and preschoolers' appetite regulation. These limitations are balanced by the fact that this study is one of the first investigations of prenatal determinants of objectively measured appetite regulation in humans, including maternal weight status prior to and during pregnancy, in a US study population spanning two critical periods of early childhood: toddler and preschool age.

\section{5 | CONCLUSION}

Study findings partially support the hypothesis that exposures during the prenatal period may influence appetite regulation in early childhood. Among girls, higher BWz and lower BWz were associated with worse objectively measured food approach behaviour. Because appetite regulation may be an important mechanism underlying prenatally induced predispositions to obesity, environmental or behavioural strategies to counteract altered appetite regulation thus may be particularly needed to prevent obesity among high-risk children such as these. However, the current study's sex-specific findings and lack of associations between maternal characteristics and children's food approach behaviour, which are counter to many findings from the animal literature, suggest further study is needed to understand the early life aetiology of appetite regulation among humans.

\section{ACKNOWLEDGEMENTS}

We thank participants in the ABC Toddler and Preschool studies. JBH and KWB conceived and designed the study and drafted the manuscript. HMW and JS conducted statistical analysis. ALM and JCL conceptualized and designed the parent study and provided critical review of the manuscript. All authors reviewed and approved the final manuscript as submitted. This research was supported by the Eunice 
Kennedy Shriver National Institute of Child Health and Human Development (R01HD069179) (PI: Lumeng and Miller) and the National Institute of Diabetes and Digestive and Kidney Diseases (K01DK102857) (Boone-Heinonen).

\section{CONFLICT OF INTEREST}

No conflict of interest was declared.

\section{ORCID}

Janne Boone-Heinonen (1D https://orcid.org/0000-0002-0368-0545

Heidi M. Weeks (D) https://orcid.org/0000-0003-3467-7987

Katherine W. Bauer (D) https://orcid.org/0000-0003-3512-3994

\section{REFERENCES}

1. Skinner AC, Ravanbakht SN, Skelton JA, Perrin EM, Armstrong SC. Prevalence of obesity and severe obesity in US children, 1999-2016. Pediatrics. 2018;141(3):e20173459.

2. Wardle J, Guthrie CA, Sanderson S, Rapoport L. Development of the Children's Eating Behaviour Questionnaire. J Child Psychol Psychiatry. 2001;42(7):963-970.

3. Dabelea D, Crume T. Maternal environment and the transgenerational cycle of obesity and diabetes. Diabetes. 2011;60(7):1849-1855.

4. Gluckman PD, Hanson MA, Cooper C, Thornburg KL. Effect of in utero and early-life conditions on adult health and disease. $N$ Engl $J$ Med. 2008;359(1):61-73.

5. Ross MG, Desai M. Developmental programming of appetite/satiety. Ann Nutr Metab. 2014;64(Suppl 1):36-44.

6. Lagisz M, Blair H, Kenyon P, Uller T, Raubenheimer D, Nakagawa S. Little appetite for obesity: meta-analysis of the effects of maternal obesogenic diets on offspring food intake and body mass in rodents. Int J Obes (Lond). 2015;39(12):1669-1678.

7. Zambrano E, Nathanielsz PW. Mechanisms by which maternal obesity programs offspring for obesity: evidence from animal studies. Nutr Rev. 2013;71 Suppl 1:S42-S54.

8. Rohde K, Keller M, la Cour Poulsen L, Bluher M, Kovacs P, Bottcher Y. Genetics and epigenetics in obesity. Metabolism. 2019;92:37-50.

9. Faith MS, Pietrobelli A, Heo M, et al. A twin study of self-regulatory eating in early childhood: estimates of genetic and environmental influence, and measurement considerations. Int J Obes (Lond). 2012;36(7):931-937.

10. Lumeng JC, Fisher JO (Eds). Pediatric Food Preferences and Eating Behaviors. Academic Press; 2018:1-34, ISBN 9780128117163, https://doi.org/10.1016/B978-0-12-811716-3.00001-4

11. Reis RS, Dalle Molle R, Machado TD, et al. Impulsivity-based thrifty eating phenotype and the protective role of n-3 PUFAs intake in adolescents. Transl Psychiatry. 2016;6(3):e755.

12. Oliveira A, de Lauzon-Guillain B, Jones $L$, et al. Birth weight and eating behaviors of young children. J Pediatr. 2015;166(1):59-65.

13. Cardona Cano S, Tiemeier H, Van Hoeken D, et al. Trajectories of picky eating during childhood: a general population study. Int J Eat Disord. 2015;48(6):570-579.

14. Silveira PP, Agranonik M, Faras H, Portella AK, Meaney MJ, Levitan RD. Preliminary evidence for an impulsivity-based thrifty eating phenotype. Pediatr Res. 2012;71(3):293-298.

15. Yu Z, Han S, Zhu J, Sun X, Ji C, Guo X. Pre-pregnancy body mass index in relation to infant birth weight and offspring overweight/obesity: a systematic review and meta-analysis. PLoS ONE. 2013;8(4):e61627.
16. Derks IPM, Hivert MF, Rifas-Shiman SL, et al. Associations of prenatal exposure to impaired glucose tolerance with eating in the absence of hunger in early adolescence. Int J Obes (Lond). 2019. https://doi.org/ 10.1038/s41366-018-0296-6

17. Carnell S, Benson L, Pryor K, Driggin E. Appetitive traits from infancy to adolescence: using behavioral and neural measures to investigate obesity risk. Physiol Behav. 2013;121:79-88.

18. Migraine A, Nicklaus $S$, Parnet $P$, et al. Effect of preterm birth and birth weight on eating behavior at 2 y of age. Am J Clin Nutr. 2013;97(6):1270-1277.

19. Johnson S, Matthews R, Draper ES, et al. Eating difficulties in children born late and moderately preterm at 2 y of age: a prospective population-based cohort study. Am J Clin Nutr. 2016;103(2):406-414.

20. Oken E, Kleinman KP, Rich-Edwards J, Gillman MW. A nearly continuous measure of birth weight for gestational age using a United States national reference. BMC Pediatr. 2003;3(1):6-6.

21. Krakowiak P, Walker CK, Tancredi DJ, Hertz-Picciotto I. Maternal recall versus medical records of metabolic conditions from the prenatal period: a validation study. Matern Child Health J. 2015;19(9): 1925-1935.

22. McClure CK, Bodnar LM, Ness R, Catov JM. Accuracy of maternal recall of gestational weight gain 4 to 12 years after delivery. Obesity (Silver Spring). 2011;19(5):1047-1053.

23. Council NR. Weight Gain During Pregnancy: Reexamining the Guidelines. Washington, DC: National Academies Press; 2010.

24. Carnell S, Wardle J. Measuring behavioural susceptibility to obesity: validation of the child eating behaviour questionnaire. Appetite. 2007;48(1):104-113.

25. Wardle J, Carnell S. Appetite is a heritable phenotype associated with adiposity. Ann Behav Med. 2009;38(Suppl 1):S25-S30.

26. Domoff SE, Miller AL, Kaciroti N, Lumeng JC. Validation of the Children's Eating Behaviour Questionnaire in a low-income preschool-aged sample in the United States. Appetite. 2015;95:415-420.

27. Asta K, Miller AL, Retzloff L, Rosenblum K, Kaciroti NA, Lumeng JC. Eating in the absence of hunger and weight gain in low-income toddlers. Pediatrics. 2016;137(5):e20153786.

28. Leung CY, Lumeng JC, Kaciroti NA, Chen YP, Rosenblum K, Miller AL. Surgency and negative affectivity, but not effortful control, are uniquely associated with obesogenic eating behaviors among low-income preschoolers. Appetite. 2014;78:139-146.

29. Kuczmarski RJ, Ogden CL, Grummer-Strawn LM, et al. CDC growth charts: United States. Advance data from vital and health statistics; no. 314. Hyattsville, Maryland: National Center for Health Statistics; 2000.

30. Boone-Heinonen J, Sacks RM, Takemoto EE, et al. Prenatal development and adolescent obesity: two distinct pathways to diabetes in adulthood. Child Obes. 2018;14(3):173-181.

31. Fisher JO, Birch LL. Eating in the absence of hunger and overweight in girls from 5 to 7 y of age. Am J Clin Nutr. 2002;76(1):226-231.

32. Kral TV, Allison DB, Birch LL, Stallings VA, Moore RH, Faith MS Caloric compensation and eating in the absence of hunger in 5- to 12-y-old weight-discordant siblings. Am J Clin Nutr. 2012;96(3): 574-583.

33. McCarthy EK, Chaoimh C, Murray DM, Hourihane JO, Kenny LC, Kiely $M$. Eating behaviour and weight status at 2 years of age: data from the Cork BASELINE Birth Cohort Study. Eur J Clin Nutr. 2015;69(12): 1356-1359.

34. Webber L, Hill C, Saxton J, Van Jaarsveld CH, Wardle J. Eating behaviour and weight in children. Int J Obes (Lond). 2009;33(1):21-28. 
35. Anzman-Frasca S, Ventura AK, Ehrenberg S, Myers KP. Promoting healthy food preferences from the start: a narrative review of food preference learning from the prenatal period through early childhood. Obes Rev. 2018;19(4):576-604.

36. Yajnik CS. Transmission of obesity-adiposity and related disorders from the mother to the baby. Ann Nutr Metab. 2014;64(Suppl 1):8-17.

37. Carpenter T, Grecian SM, Reynolds RM. Sex differences in early-life programming of the hypothalamic-pituitary-adrenal axis in humans suggest increased vulnerability in females: a systematic review. J Dev Orig Health Dis. 2017;8(2):244-255.

38. Jeanne TL, Hooker ER, Nguyen T, et al. High birth weight modifies association between adolescent physical activity and cardiometabolic health in women and not men. Prev Med. 2018;108:29-35.

39. Cheong JN, Wlodek ME, Moritz KM, Cuffe JS. Programming of maternal and offspring disease: impact of growth restriction, fetal sex and transmission across generations. J Physiol. 2016;594(17): 4727-4740.

40. van Deutekom AW, Chinapaw MJ, Vrijkotte TG, Gemke RJ. The association of birth weight and postnatal growth with energy intake and eating behavior at 5 years of age-a birth cohort study. Int J Behav Nutr Phys Act. 2016;13(1):15.
41. Yu ZB, Han SP, Zhu GZ, et al. Birth weight and subsequent risk of obesity: a systematic review and meta-analysis. Obes Rev. 2011;12(7):525-542.

42. Li A, Teo KK, Morrison KM, et al. A genetic link between prepregnancy body mass index, postpartum weight retention, and offspring weight in early childhood. Obesity (Silver Spring). 2017;25(1): 236-243.

43. Connor Gorber S, Tremblay M, Moher D, Gorber B. A comparison of direct vs. self-report measures for assessing height, weight and body mass index: a systematic review. Obes Rev. 2007;8(4):307-326.

44. Headen I, Cohen AK, Mujahid M, Abrams B. The accuracy of self-reported pregnancy-related weight: a systematic review. Obes Rev. 2017;18(3):350-369.

How to cite this article: Boone-Heinonen J, Weeks HM, Sturza J, Miller AL, Lumeng JC, Bauer KW. Prenatal predictors of objectively measured appetite regulation in low-income toddlers and preschool-age children. Pediatric Obesity. 2019;14:e12554. https://doi.org/10.1111/ijpo.12554 